\title{
Correction to: Transferring the sandwich principle to instructional videos: is it worth the effort?
}

\author{
Anna Bock ${ }^{1 *}$, Christina Thomas ${ }^{1}$, Marius Heitzer ${ }^{1}$, Philipp Winnand ${ }^{1}$, Florian Peters ${ }^{1}$, Martin Lemos ${ }^{2}$, \\ Frank Hölzle ${ }^{1}$ and Ali Modabber ${ }^{1}$
}

\section{Correction to: BMC Med Educ 21, 525 (2021)}

https://doi.org/10.1186/s12909-021-02967-3

Following publication of the original article [1], we have been informed that the given name and family name were erroneously transposed for all the authors.

The author group has been updated above and the original article [1] has been corrected.

\section{Author details}

'Department of Oral and Maxillofacial Surgery, University Hospital RWTH

Aachen, Pauwelsstrasse 30, 52074 Aachen, Germany. ${ }^{2}$ Audiovisual Media Center, Medical Faculty, RWTH Aachen University, Pauwelsstraße 30,

52074 Aachen, Germany.

Published online: 07 January 2022

\section{Reference}

1. Bock A, et al. Transferring the sandwich principle to instructional videos: is it worth the effort? BMC Med Educ. 2021;21:525. https://doi.org/10.1186/ s12909-021-02967-3. 